\title{
Hyperelastic modelling of nonlinear running surfaces
}

\author{
R. D. Thomson, A. E. Birkbeck and T. D. Lucas \\ Department of Mechanical Engineering, University of Glasgow, Scotland and Adidas-Salomon AG, Germany
}

\begin{abstract}
Accurate, 3-D analyses of running impact require a constitutive model of the running surface that includes the material nonlinearity shown by many modern surfaces. This paper describes a hyperelastic continuum that mimics the experimentally measured response of a particular treadmill surface. The material model sacrifices a little accuracy to admit a robust, low-order hyperelastic strain-energy functional. This helps prevent the premature termination of finite element simulations, due to numerical or material instabilities, that can occur with higher-order functionals. With only two free constants, it is also a more practical design tool. The best fit to the quasi-static response of the treadmill was achieved with an initial shear modulus $\mu=2 \mathrm{MPa}$ and a power-stiffening index $\alpha=-25$. The paper outlines the method used to derive the material constants for the treadmill, a device that is not amenable to the usual materials laboratory tests and must be reverse-engineered. Finite element analyses were then performed to ensure that the treadmill model interacts with the other components of the multibody running system in a numerically stable and physically realistic manner. The model surface was struck by a rigid heel, cushioned by a hyperfoam material that represents a shoe midsole. The results show that, while the ground reaction force is similar to that obtained with a rigid surface, the maximum principal stress in the shoe is reduced by $15 \%$. Such a reduction, particularly when endured over many load cycles, may have a significant effect on comfort and damage to nearby tissue.
\end{abstract}

Keywords: running surface, injury, damage, hyperelasticity, finite elements

\section{Introduction}

In the design of sports equipment for a market that is worth over $£ 3$ billion annually in the UK alone (Taylor 1998), the drive for performance must be tempered by the need for athletes' comfort and for protection against sports injuries that cost both individuals and medical services. Damage resulting from the repeated impact of an

Correspondence address:

R. D. Thomson, Department of Mechanical Engineering, University of Glasgow, Scotland.

Tel.: 0141-330-4313. E-mail: r.thomson@mech.gla.ac.uk athlete's foot on a running surface is particularly common, especially amongst leisure runners who are unlikely to be coached and who often overstretch their abilities. However, while there is a significant body of literature on the effects of shoe and surface materials in running impact, most of this work has been led by life scientists or clinicians primarily interested in physiology, biology, etc. or the treatment of sports injuries. The contribution of engineering stress analysis to the design of products that can reduce the risk of such injuries has, so far, been minor. However, this is changing as an understanding of how structural and material factors affect function and performance is increasingly recognized as a prerequisite for the design of innovative and exciting products. 
Of course, applying thermomechanical principles to systems as complex as athletes and their equipment, in a way that gives theoretical insight or aids in product design, is problematic. However recent developments in large-deformation constitutive theories, damage mechanics, multibody system dynamics and computational methods allow increasingly realistic analysis of such systems, feeding the growth of what is now called 'sports engineering'.

\section{System modelling}

It is well recognized that athletes interact with their equipment in an individual and subjective manner and, in running, individual gait may be the dominant aspect of the whole system (Ferris et al. 1999). However this knowledge is of little use to manufacturers and designers who require reliable, objective, quantitative means of predicting the performance of new equipment in a whole tranche of the population. Mathematical models of shoes and running surfaces are an essential feature in such work and, while the details of materials and construction will determine the performance of any particular system, it is initially appropriate to investigate generic models that are more broadly applicable, and to develop means of generating the data needed for analyses. It might be more satisfying to be able to predict the mechanical behaviour of products before they are manufactured but, in reality, it is often necessary to reverseengineer existing products in order to discover why particular, often poorly understood features have proven successful or otherwise.

\section{Continuum models}

Over the last 20 years, from the seminal work of McMahon \& Greene (1979) to more recent studies by Dalleau et al. (1998), many authors have developed and used lumped-parameter models to describe the locomotion of runners and to predict the performance of equipment. These models continue to be useful and can be extended to include some nonlinearities. However, it is increasingly apparent
(Lemmon et al. 1997; Thomson et al. (1999), Miller et al. (2000)) that a continuum approach such as nonlinear finite element analysis (NL FEA) offers more scope for modelling the complex structural details and the sophisticated materials used in modern sports equipment. This is particularly true when the equipment is subject to service loadings that are difficult to replicate in laboratory experiments but can be easily simulated computationally. The current work therefore aims to develop a material model of a particular running surface, in this case a treadmill, that can be incorporated into more realistic 3-D FE analyses of running.

FEA is a sophisticated tool but it is an approximate method with sources of error that are inherent in the formulation and not simply a result of its implementation on digital computers. In particular, the 'displacement method' of stress analysis, which is most commonly implemented in commercial FE codes, uses low-order polynomials and often just linear functions to interpolate the displacement between the nodes in an element. This gives an upper bound on the stiffness of the real structure. The inevitable error in the results can be reduced, up to a point, by refining the mesh. However no amount of mesh refinement or even adaptive meshing can compensate for an inadequate material model. Indeed, an inappropriate choice of constitutive behaviour or simply erroneous data may completely invalidate the analysis, even if the actual specification of the material occupies only a few lines of an input file containing thousands of lines of code. This is particularly true if the material response diverges from linearity at small strains, as is the case with the polymers commonly used in modern running shoe cushioning and in synthetic running surfaces. In an attempt to address this, D'Agati \& Ladin (1993) used a bilinear approximation to model the response of shoe cushioning material. However they rightly noted the difficulties in such a piecewise approach which is, in any case, difficult to translate into useful design rules. It is then better to consider continous functions that admit material nonlinearity. 


\section{Hyperelasticity}

Hyperelastic materials, typically rubber-like polymers, belong to a class of material that is important, not only in sports engineering, but in applications ranging from vehicle suspensions to artificial heart valves. This is mainly because they can be easily formed, for example by compression moulding, into monolithic components that exhibit sophisticated force-deformation behaviours. The essential feature in the constitutive model of a hyperelastic material is the existence of a sufficiently continuous strain-energy functional $U$ that acts as a potential from which stresses may be derived by differentiation with respect to a kinematic quantity, such as the stretch ratio $\lambda$ (Ogden 1987). Hyperelastic materials need not be nonlinear, although most are, but they are path-independent and do not admit hysteresis losses. Such losses can be a significant proportion of the strain energy stored in the shoe (Thomson et al. 1999) but they are a small fraction of the total energy cost of running and hyperelasticity is a useful approximation to the constitutive response of many of the materials used in running and other sports. Truly rubber-like materials are almost incompressible, having a very high bulk modulus or, equivalently, a Poisson's ratio $v \cong 0.5$, but hyperelastic constitutive theory has been extended to admit compressibility, giving what is known as a hyperfoam (Storakers 1986). Both hyperelasticity and hyperfoam behaviour have been encoded into several industry-standard NL FEA codes, including Abaqus/Explicit (Version 5.8) (HKS 1998) as used in the current work. In the Abaqus hyperfoam formulation:

$$
\begin{aligned}
\mathrm{U}= & \sum_{\mathrm{i}=1}^{\mathrm{N}} 2 \times \mu_{\mathrm{i}} / \alpha_{\mathrm{i}}^{(2)} \\
& \times\left[\lambda_{1}^{\left(\alpha_{\mathrm{i}}\right)}+\lambda_{2}^{\left(\alpha_{\mathrm{i}}\right)}+\lambda_{3}^{\left(\alpha_{\mathrm{i}}\right)}-3+1 / \beta_{\mathrm{i}} \times\left(\mathrm{J}_{\mathrm{el}}^{-\alpha_{\mathrm{i}} \beta_{\mathrm{i}}}-1\right)\right]
\end{aligned}
$$

where the $\lambda_{\mathrm{i}}$ are the principal deviatoric stretches, $\alpha_{i}, \mu_{i}$ and $\beta_{i}$ are material constants and $N$ is the 'order' of the model. The $\mu_{\mathrm{i}}$ are material stiffnesses and their summation is equal to the initial shear modulus. The $\alpha_{\mathrm{i}}$ are undimensioned constants that control the curvature of the elastic response and so determine the rate of tension or compression stiffening.

Hyperelastic modelling of the materials typically used for running equipment or in similar applications is still fairly novel, and material property data remains scarce. Lemmon et al. (1997) used a hyperelastic material model of soft tissue and a third-order hyperfoam model of the insole and midsole to study the effects of sole cushioning in therapeutic footwear. However this requires the determination of 25 material constants before it even addresses the structural and geometric complexities, such as encapsulated fluid, that feature in modern running shoes. It is thus too complex to be of use in product design engineering. Of course, running shoes, surfaces and athletes are subject to 3-D loading but the material constants needed to model their deformation are usually determined in simpler loading conditions, such as uniaxial tension, that can be reproduced in controlled laboratory experiments. Using these constants in more general loading states is often justified more by practical experience than by mathematical rigour, but it is often the only option available to designers and simulation engineers. In the case of hyperelastic materials, even the 'simple' laboratory experiments needed to determine the full nonlinear-elastic constitutive tensor are not trivial, and may require uniaxial, biaxial, planar and volumetric deformations (Ogden 1984; Abaqus/Explicit User Manual Vol 1 (HKS 1998)). Indeed, many sports engineering materials, such as the EVA foam that is often used in shoe cushioning and the rubber-crumb matting that is often used for athletic surfaces, have a high volume fraction of air-filled and possibly opencelled cavities. The response of such voided materials is not only nonlinear and time-dependent but is even dependent on the sign of the hydrostatic component of the stress tensor. In essence, tensile loading causes stretching of the cavity walls while compression causes elastic buckling and collapse of the cavity, ultimately leading to what runners know as 'bottoming out'. Given laboratory-scale material samples, this pressure-sensitive response can be determined (Miller, 
Baroud \& Nigg (2000); Mills \& Gilchrist (2000)). However structures such as treadmills, and even shoe features such as encapsulated fluid, which are also nonlinear-elastic, are not so amenable to standard laboratory tests and may have to be reverse-engineered.

\section{Shoe cushioning model}

Fortunately, there are factors that mitigate the difficulty in materials modelling in particular cases such as running sports. Unlike many other sports, the impact loads on running equipment are predominantly compressive and a full hyperelastic test programme may not be necessary to infer a material model that yields useful results. This is particularly true when the low Poisson's ratio that results from a high void-volume fraction in foamlike materials makes the stress state relatively insensitive to the nature of the transverse constraints. Adopting the practice used in continuum damage mechanics (CDM), by which a material with a heterogeneous microstructure is modelled as a homogeneous continuum, Thomson et al. (1999) developed first-order hyperfoam models that are a good fit to the aggregate response of modern training shoe cushioning systems, including encapsulated-air soles. That study used a shear modulus $\mu=0.2 \mathrm{MPa}$, a Poisson's ratio $v=0.2$ and hyperelastic power-stiffening indexes of $\alpha=1.8$ for encapulated-air shoes and $\alpha=1.7$ for solid EVA soles. In the current work, the value of $\alpha=1.8$ is adopted as an exemplar. A previously developed mesh (Fig. 1, upper block) of 4-noded axisymmetric isoparametric quadrilateral continuum elements (Abaqus type CAX4R) was used to give a force-displacement response, under quasi-static compressive loading, that is typical of modern running shoes. To simulate the constraining influence of the outer material on the midsole, the right-hand edge of the shoe model was allowed to move in the 2-direction only. However the analysis showed that this boundary condition does not have a significant effect on the deformation of the foam except, of course, near this outer edge.

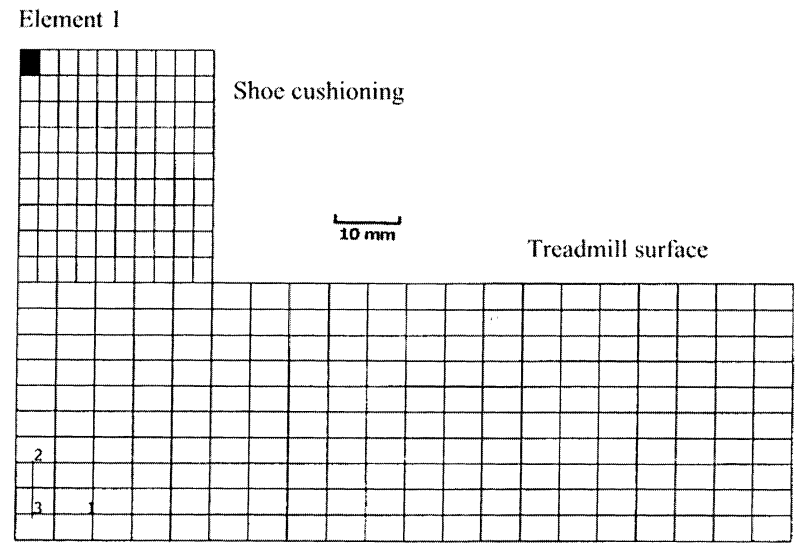

Figure 1 The finite element mesh. The upper block represents the running shoe cushioning and the lower block the treadmill surface.

Treadmill surface model

To support physiological studies of the effect of surface compliance on running economy, Grant et al. (1998) measured the quasi-static force-deflection response of two different treadmill running surfaces; both were found to be nonlinear-elastic. Baroud et al. (1999) chose a viscoelastic material model in studies of energy return from running surfaces but Walker (1998) has argued that the time constants of the materials studied are too large for viscosity to play any role in the short-duration impacts typical in running. In addition, the duration of the stance phase of running is always of the order of a few tenths of a second. The ratecontrolled stiffening that is a necessary feature of polymeric constitutive models designed to cover a wide range of strain rates, can then be factored out of a footstrike simulation. A nonlinear hyperelastic model would then seem to be appropriate for nonplastic running surfaces such as treadmills and synthetic tracks.

The treadmill surfaces tested by Grant et al. (1998) are nonlinear-elastic and the difference in stiffness is important. However the bulk modulus, even if this could defined for such a machine, is not of interest for future work that will focus on the running shoes. The surface can then be modelled as hyperelastic rather than hyperfoam, with the mesh shown in Fig. 1 (lower block). This was also built 
from CAX4R elements. Physically, treadmill surfaces usually comprise a continuous loop of flexible, rubber mat supported on a stiff composite or wooden deck driven by an electric motor via rollers. The 'Woodway' machine modelled here is somewhat different in that it has an articulated loop of transverse, simply supported slats made of a stiff polymer (Fig. 2). The surface response of such a machine is not readily inferred from materialslaboratory test specimens. Of course a complex finite element model of the slats and support frame could be built but the construction details are also irrelevant here; what is essential is that the model generates a footstrike reaction that is similar to that of the real treadmill.

The key property to be modelled here is the structural stiffness of the machine, an extrinsic property defined as the ratio of applied force to displacement. However, hyperelastic material characteristics must be specified to Abaqus in the form of the intrinsic material properties. These may be input directly, as values of $\alpha, \mu$, etc., or indirectly, in the form of a table of stress-strain pairs from which these constants can be calculated. Such data is usually derived from standard laboratory tests but, as noted previously, this is not practical for a treadmill machine. As with the shoe cushioning, aggregate values, describing the overall structural response of the treadmill surface, were therefore sought by a more pragmatic approach. Initially, a table of starting values for stress and strain was entered and the model's force-displacement response under increasing quasi-static load compared to the actual response of the real treadmill.

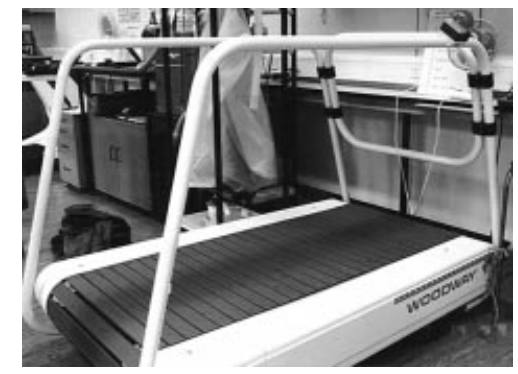

Figure 2 The 'Woodway' treadmill. The slatted construction of the running surface is apparent.
The stress and strain values were then incrementally adjusted in a series of computational experiments, until the best fit to the experimental data was obtained (Fig. 3). The starting values of stress and strain can be almost arbitrary but, obviously, the rate of convergence to the desired curve is greater if initial values, of the right order of magnitude, can be estimated from past experience with similar materials. For the best fit, with firstorder Ogden hyperelasticity, Abaqus returned the values $\mu=2 \mathrm{MPa}$ and $\alpha=-25$. An improved fit could have been obtained with a second or third order model but this introduces more free constants and reduces the utility of the model as a design tool. More seriously, higher order models tend to lead to numerical or material instabilities at large strains. This is discussed later.

In the course of the computational experiments, several combinations of boundary conditions were tried to obtain a match to the experimental behaviour of the treadmill. Supporting only the right-hand edge of the treadmill nodes gave an

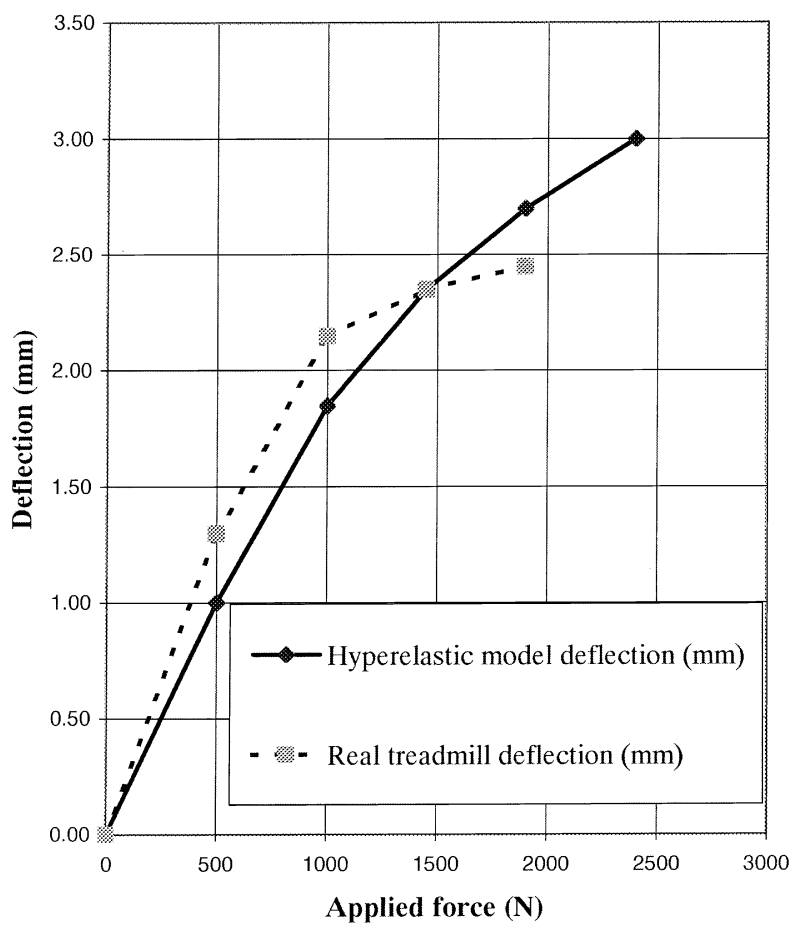

Figure 3 Modelling the response of the 'Woodway' treadmill surface as a first-order hyperelastic material. 
almost-linear beam-like response even with a nonlinear stress-strain law and so foundation support was provided, with the base nodes constrained to move only in the 1-direction.

\section{Multi-body impact model}

Both the running surface model and the shoe cushioning model were based on quasi-static experimental data. These models must obviously interact in a multibody dynamic simulation of impact that yields results that are both numerically stable and physically realistic, in that they are consistent with well-known experimental results.

As previously noted, it is also necessary to test the FE model for material or numerical instabilities to the level of deformation to be expected in service. These instabilities can cause the analysis to abort before any useful results have been obtained and there is little alternative here other than to run a simulation of the complete duration of the expected event, adjusting the input data and the analysis defaults as required. This requires an extensive series of computational experiments. Thus, while the current axisymmetric models of the shoe cushioning and the treadmill surface cannot fully represent a footstrike, they are more suitable than a full 3-D simulation for numerical testing of material models.

The model shoe and treadmill were then concatenated into a single multibody system. The undeformed assembly is shown in Fig. 1, in which there is a very small initial gap between the shoe mesh and the treadmill mesh, to ensure no initial overclosure. As in previous quasi-static analyses by Thomson et al. (1999), the runner's heel was simulated by a rigid surface, which does not admit stress recovery in its interior. This is not a problem for the current work but a full mesh would be required for study of the loads in the heel itself. The impact analyses do however, require the runner's body weight to be represented. A point mass element of $70 \mathrm{~kg}$, on which gravitational loads were imposed, was therefore placed on the centreline above the heel and connected to it by a spring. For this material-development phase of the work, a single linear spring with a stiffness of $16 \mathrm{kN} \mathrm{m}^{-1}$ (Farley \& Gonzalez 1996) was chosen to represent what is, in reality, a complex biomechanical linkage of variable stiffness. The length of the legspring element is not critical for purely vertical impact but it must not be so short as to cause it to invert during the motion - a physically impossible situation that can occur numerically. A length of $1 \mathrm{~m}$ was therefore used, conveniently placing the mass element near the centre of mass of a real runner.

Both the heel and the point mass were assigned an initial velocity of $1.2 \mathrm{~m} \mathrm{~s}^{-1}$ downwards, the vertical component of the footstrike velocity as measured by high-speed digital video recordings of a runner. Assigning the same initial vertical velocity to all of the nodes in both the shoe and leg subsystems, both of which are then subject to gravitational acceleration, prevents deformation of the shoe sole prior to impact. Abaqus/Explicit was instructed to simulate $0.3 \mathrm{~s}$ of real time and the defaults on the contact algorithm were over-ridden to force the contact nodes on the low-density shoecushioning material to be pure slaves. Adaptive meshing was also enabled in the highly compliant shoe-cushioning elements to prevent excessive shear distortion of the quadrilaterals, a common occurrence that degrades the accuracy of any FEA and can ultimately cause the run to abort. In the version of Abaqus used here, the rezoning algorithm changes the shape of poor-quality elements but does not increase the mesh density in regions of high deformation gradients. Again the defaults had to be over-ridden to admit rezoning on every deformation increment and to monitor every element shape individually. This drastically increases the analysis time but was necessary to allow the simulation to run to completion.

\section{Results and discussion}

A first-order hyperelastic model, with $\mu=2 \mathrm{MPa}$ and $\alpha=-25$ was found to adequately represent the experimental response of the real treadmill and allow simulation, without numerical problems, of the duration of running impacts. This simulation, 
of $300 \mathrm{~ms}$ of real-time, required $70 \mathrm{~min}$ of CPUtime on a Unix machine.

Figure 4 shows the progressive deformation of the treadmill surface and the shoe cushioning, the indentation due to the heel being apparent. The deformation is magnified here, to confirm that the treadmill surface does indeed move, the actual compression of the shoe being $20 \mathrm{~mm}$ at a load of $2 \mathrm{kN}$ (Thomson et al. 1999). Both of the subsystems shown, and the mass, heel and legspring that have been omitted for clarity, appear well-behaved, the plots showing no evidence of pathological events, such as material interpenetration, that can occur in FEA of highly nonlinear events such as contact.

Figure 5 shows the development of the ground reaction force (GRF) for both the hyperelastic treadmill surface and for an almost-rigid surface.
There is some numerical chatter in the curves but they are generally well-behaved, the overall shape and the peak value of about $2 \mathrm{kN}$, approximately three times body-weight, being in reasonable accord with well-known experimental results (e.g. Whittle (1997)). The contact time of $300 \mathrm{~ms}$ is also not unreasonable (e.g. Ferris et al. (1999)) and admits comparison of the effect of the running surface stiffness. Of course, both the peak GRF and the contact time depend on the compliance of the whole system and the chosen leg-spring stiffness of $16 \mathrm{kN}$ also appears satisfactory. Indeed, analyses in which this value was significantly different failed due to numerical problems. Changing the stiffness of the shoe material would also result in a different GRF and contact time but the treadmill properties, being derived from static analyses, are not dependent on either the legspring or shoe properties.

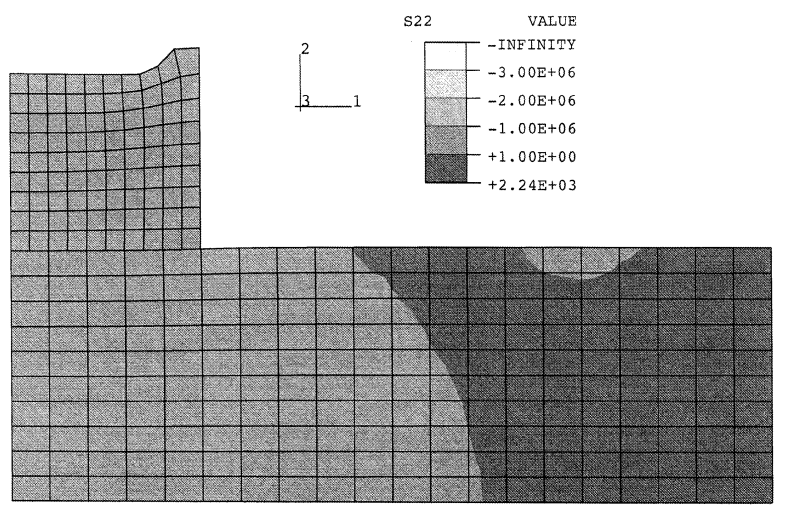

(a)

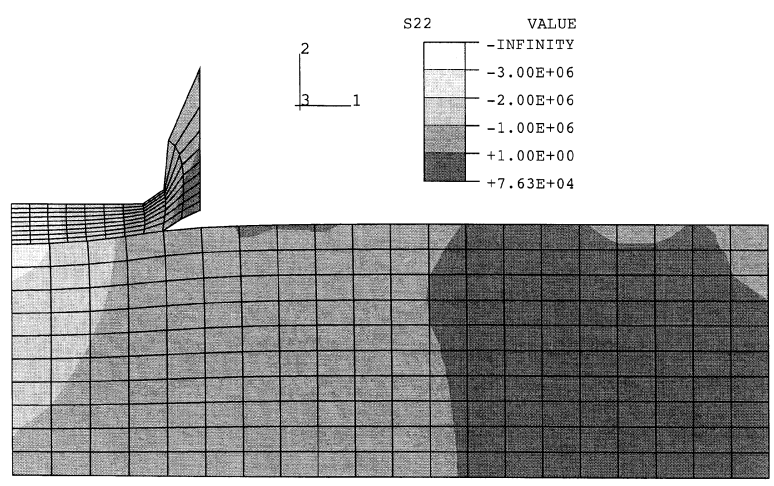

(c)

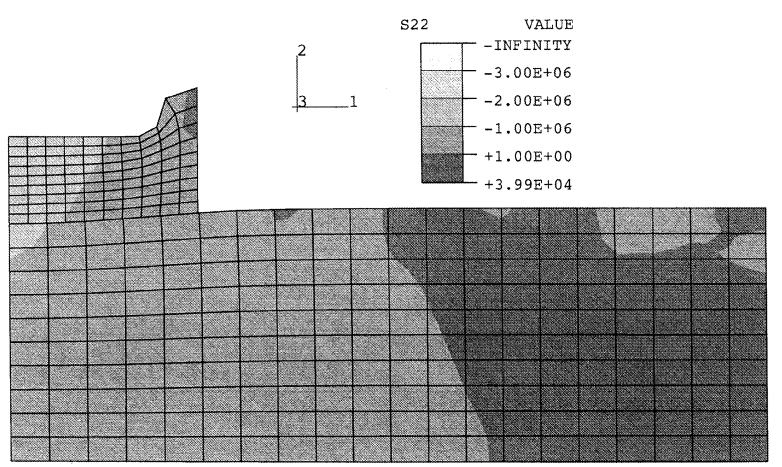

(b)

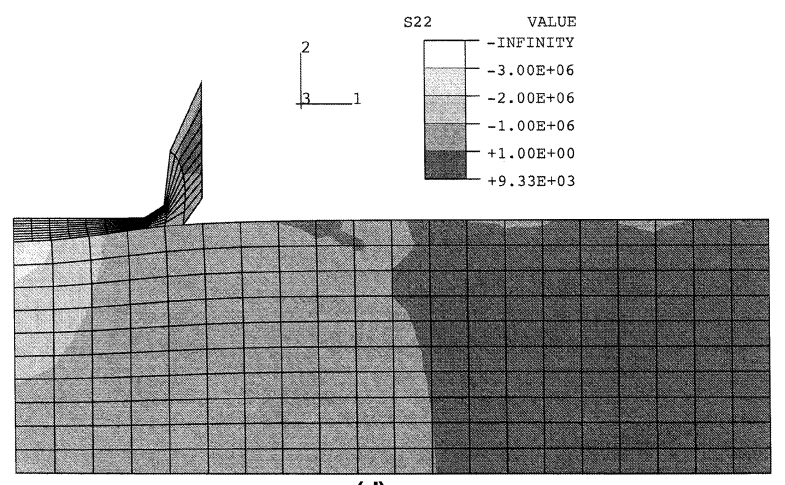

(d)

Figure 4 Progressive deformation of the system under impact loading. S22 is the vertical component of the Cauchy stress tensor. 

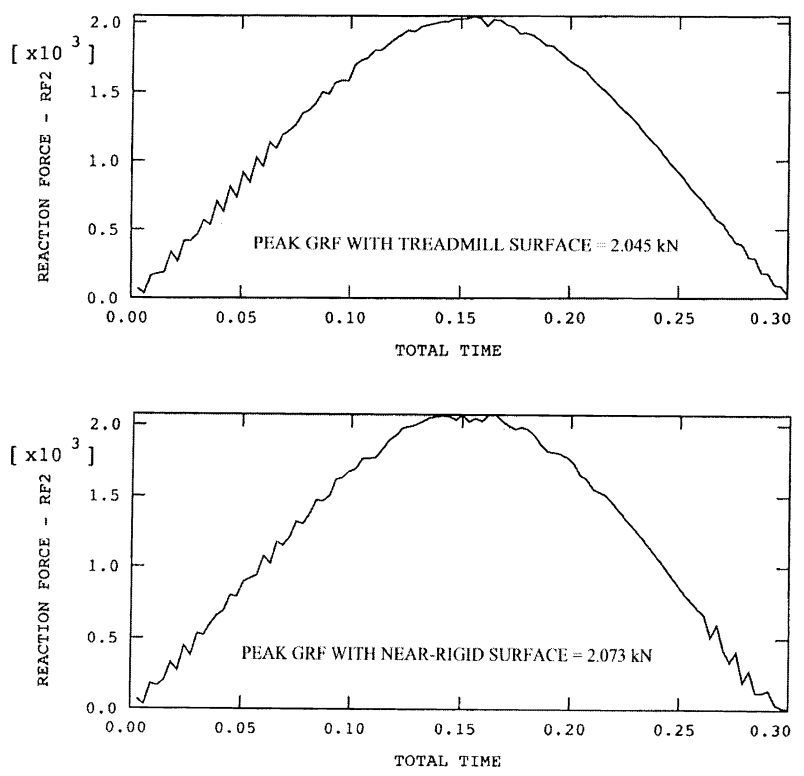

Figure 5 The predicted ground reaction force for the treadmill model and for a rigid surface.

The computed GRF does not predict the initial peak that force-plate measurements detect shortly after heelstrike. This is not surprising since this initial peak is a consequence of the mechanics of the leg linkage and not of the material properties of either running surface or shoe. It can be reproduced computationally, even in the axisymmetric model considered here, but discussion of this is outside the scope of the current work. Here it is more important to note that the GRF for both the hyperelastic surface and for a near-rigid surface are almost identical. However experienced runners (including the first author) agree that a more-compliant surface such as the treadmill is easier on the feet than is a near-rigid surface. However pain and mechanical damage to human tissue and other materials is caused by locally high stresses rather than force as such, as simple indentation experiments with increasingly sharp objects confirm, and so the stress in the region of the shoe that is in contact with the heel may be a better indicator than GRF of the risk of damage and injury. The 22-component of the Cauchy stress tensor, that is also shown in Fig. 4, is the maximum principal (compressive) stress and Fig. 6
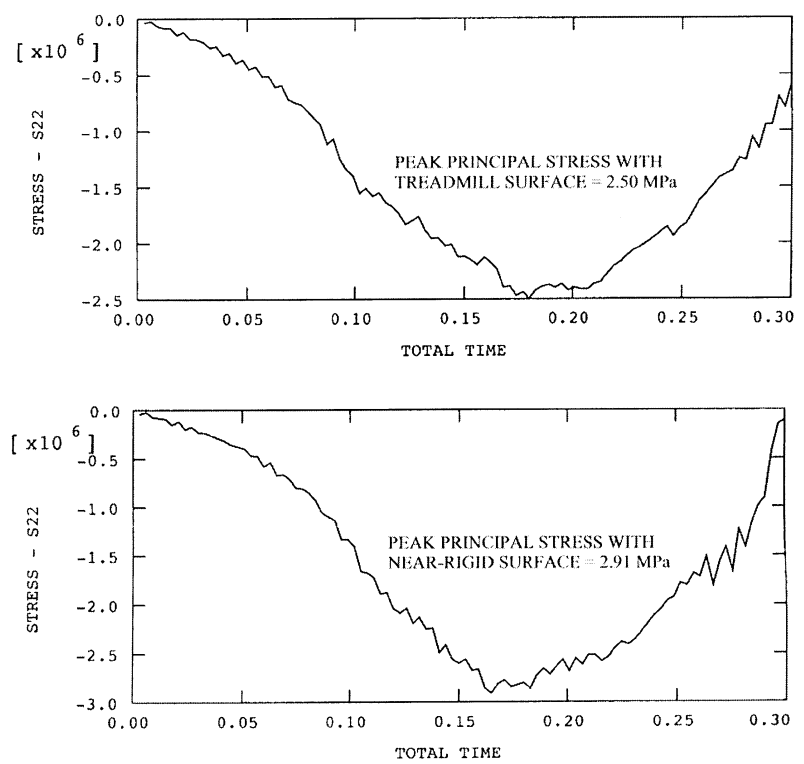

Figure 6 The maximum principal stress in the most severely loaded element (element 1).

plots its evolution in the most highly stressed element (Fig. 1, element 1) for both the treadmill and a rigid surface. Such compression is not damaging, at least at moderate levels, in static systems and in systems with no residual stresses but running is certainly not a static event and the presence of residual stresses in either the equipment or human hard tissue cannot be discounted. This points towards areas for future study. Here it may be noted that the treadmill surface induces a peak maximum principal stress in the shoe, where it contacts the heel, that is only $85 \%$ of that produced by a rigid surface. This may account for the runners' perceptions. The numerical difference might be even more pronounced with a more realistic heel profile than is used here and it would be a useful reduction even in a single load cycle. However, it is of greater significance in a sport like running, where it would be repeated on each of the several thousand load cycles that are endured on even a short run, and the system is subject to high-cycle fatigue loading. In such circumstances, damage can initiate and propagate at stresses well below the single-cycle overload stress. Again this points towards future work. 


\section{Conclusions}

The aim of the current work was to develop a model of a real running surface, in this case a treadmill machine, that will be of use in more detailed, 3-D models to aid in the engineering design of running shoes. The homogeneous continuum approach adopted here, and adapted from CDM, allows the essential features of the treadmill response to be represented, and the shoe behaviour to be studied, without the computational and experimental cost of modelling the constructional details of the machinery.

The first-order hyperelastic material model used here limits the number of free constants to a manageable number and minimizes the risk of the material or numerical instabilities that can cause the simulation to abort prematurely. Here, $\mu=2 \mathrm{MPa}$ and $\alpha=-25$ reproduce the behaviour of a 'Woodway' treadmill and allow a successful simulation of $300 \mathrm{~ms}$ of real-time.

The method of determining these material properties could be used for many other types of running surface or machine, particularly those from which laboratory material specimens cannot be obtained or in which the surface is sprung in a mechanically complex manner.

That the material models developed from static physical and computational experiments lead to dynamic simulations that are numerically robust and give results in accord with physical experiments is encouraging. This suggests that nonlinear hyperelasticity is an appropriate material model and it is anticipated that its increasing use will allow different approaches to modelling to be compared.

The subjective view of runners, that the treadmill is easier on the feet than a more rigid surface, is borne out by examination of the maximum principal stress rather than by the GRF. This reinforces the view that a continuum approach such as NLFEA, that admits nonuniform stress fields, is needed for the development of advanced products that can reduce the risk of injury.

\section{Acknowledgements}

Thanks are due to a number of exchange students who undertook some of the extensive computational experiments that have been distilled into this paper, in particular Xavier Rodriguez de Tembleque Herruzo and Tan Han Ming.

\section{References}

Baroud, G., Nigg, B.M. \& Stefanyshyn, D. (1999) Energy storage and return in sport surfaces. Sports Engineering, 2, 173-180.

D'Agati, M.C. \& Ladin, Z. (1993) A nonlinear analysis of a running shoe midsole. In Proceedings of the XIVth Congress of the International Society of Biomechanics. pp. 302-303. Societe de Biomechanique, Paris.

Dalleau, G. et al. (1998) The spring-mass model and the energy cost of treadmill running. European fournal of Physiology, 77, 257-263.

Farley, C.T. \& Gonzalez, O. (1996) Leg stiffness and stride frequency in human running. Fournal of Biomechanics, 29, 181-186.

Ferris, D.P., Liang, K. \& Farley, C.T. (1999) Runners adjust leg stiffness for their first step on a new running surface. Fournal of Biomechanics, 32, 787-794.

Grant, S. et al. (1998) The effect of the engineering properties of treadmill surfaces on the performance of athletes in a sub-maximal test. Sports Engineering, 1, 57-62.

Hibbitt, Karlsson, \& Sorenson (1998) Abaqus/Explicit Manuals. HKS Inc, Pawtucket, RI, USA.

Lemmon, D. et al. (1997) The effect of insoles in therapeutic footwear - a finite element approach. Fournal of Biomechanics, 30, 615-620.

McMahon, T. A. \& Greene, P.R. (1979) The influence of track compliance on running. Fournal of Biomechanics, 12, 893-904.

Miller, J.E., Baroud, G. \& Nigg, B.M. (2000) Elastic behaviour of sport surface materials. Sports Engineering, 3, 177-184.

Mills, N.J. \& Gilchrist, A. (2000) Modelling the indentation of low-density polymer foams. Cellular Polymers, 19, 389-412.

Ogden, R.W. (1984) Non-Linear Elastic Deformations. Ellis Horwood, Chichester, UK.

Storakers, B. (1986) On material representation and constitutive branching in finite compressible elasticity. Journal of the Mechanics and Physics of Solids, 34, 125-145.

Taylor, P. (1998) The economics of the sports products industry. In: The Engineering of Sport: Proceedings of the 
Hyperelastic modelling of nonlinear running surfaces - R. D. Thomson et al.

2nd International Conference on Sports Engineering

(ed. S. J. Haake), pp. 171-179. Blackwell Science, Oxford, UK.

Thomson, R.D. et al. (1999) The modelling and performance of training shoe cushioning systems. Sports Engineering, 2, 109-120.
Walker, C.A. (1998) A hybrid system for testing training shoes. In The Engineering of Sport: Proceedings of the 2nd International Conference on Sports Engineering (ed. S. J. Haake), pp. 109-113. Blackwell Science, Oxford, UK. Whittle, M.W. (1997) Gait Analysis. Butterworth-Heinemann Medical, Oxford, UK. 